\title{
Comparison of a purely endoscopic three-layer technique versus pericranial flap for reconstruction of anterior skull base defects after sino-nasal tumor resection: assessment of postoperative frontal lobe sagging and frontal lobe
} falling*

\author{
Giacomo Fiacchini', Silvia De Santi', Domenico Tricò ${ }^{2}$, Christina Cambi', \\ Veronica Seccia ${ }^{3}$, Lodovica Cristofani-Mencacci', Stefano Berrettini', Luca \\ Bruschini', Mariella Scarano', lacopo Dallan'
}

Rhinology 58: 5, $482-488,2020$

https://doi.org/10.4193/Rhin19.431

\section{*Received for publication:}

November 30, 2019

Accepted: March 2, 2020

Department of Surgical, Medical, Molecular Pathology and Emergency Medicine, University of Pisa, Pisa, Italy

2 Department of Clinical and Experimental Medicine, University of Pisa, Pisa, Italy

${ }^{3}$ First ENT Unit, Azienda Ospedaliero-Universitaria Pisana, Department of Surgical, Medical, Molecular Pathology and Emergency

Medicine, University of Pisa, Pisa, Italy

\begin{abstract}
Background: The evolution of endoscopic skull base approaches has enabled surgeons to manage selected skull base tumors through a transnasal endoscope-assisted approach. On the other side, more extensive lesions may require a combined cranioendoscopic approach. In this paper, we analysed and compared the incidence of frontal lobe sagging after endoscopic multilayer (EM) reconstruction versus pericranial flap (PF) reconstruction.
\end{abstract}

Methodology: Subjects were selected retrospectively according to specific inclusion and exclusion criteria. The degree of frontal lobe sagging after surgery was calculated based on the most inferior position of the frontal lobe relative to the nasion-sellar line defined on preoperative and postoperative imaging. A positive value signified upward displacement, and a negative value represented frontal lobe sagging.

Results: Twenty subjects were enrolled in our study. In the EM technique group the average frontal lobe displacement was -2,34 $\pm 1,55 \mathrm{~mm}$. The average postoperative frontal lobe sagging was $-0,45 \pm 8,92 \mathrm{~mm}$ in subjects reconstructed with the PF. The skull base defect size correlated with the degree of frontal lobe sagging in subjects reconstructed with the PF, but not in the other group and when merging the two groups.

Conclusions: In conclusion, the EM technique and the PF reconstruction showed a good reliability for the closure of anterior skull base defects. Moreover the PF seemed to prevent frontal lobe sagging but, for larger skull base defects, it could be useful to be combined with other autologous or heterologous materials to avoid the frontal lobe falling.

Key words: anterior skull base defect, frontal lobe sagging, pericranial flap, skull base reconstruction, three-layer technique

\section{Introduction}

The evolution of endoscopic skull base approaches has enabled surgeons to manage selected skull base tumors through a pure transnasal endoscope-assisted approach. On the other side, more extensive lesions may require a combined cranio-endo- scopic approach.

The experience acquired up to date is absolutely significant, and data from literature demonstrate the validity of the endonasal approach in the management of sinonasal and anterior skull base tumours ${ }^{(1)}$. But it must be stressed that endoscopic assisted 
procedures are not riskless ${ }^{(2)}$. Among possible postoperative complications, one of the less investigated is the frontal lobe sagging that could be associated with other major problems like brain herniation, cerebrospinal fluid (CSF) leak or, if the frontal lobe slides into the radiation field during adjuvant radiotherapy, to cerebral necrosis.

In this paper, we analysed and compared the incidence of frontal lobe sagging after endoscopic multilayer (EM) reconstruction versus pericranial flap (PF) reconstruction in subjects submitted to resection of anterior skull base tumors. To the best of our knowledge, this is the second paper ${ }^{(3)}$ that analyses this postoperative outcome but it is the first that compares it in two different surgical techniques. Moreover we identified and defined a particular frontal lobe sagging that we called frontal lobe falling.

\section{Methods}

Subjects were selected retrospectively through our digital database (Ormaweb, Avelco SRL) according to the following inclusion and exclusion criteria.

Inclusion criteria: an age of at least 18 years old; only one craniotomy performed in subjects' lifetime; reconstructive procedures allowed are only EM technique and PF; surgery performed from June 1st 2012 to December 31st 2018; minimum 1-month follow-up; benign or malignant neoplasm at final histology. Exclusion criteria: subjects who had undergone two or more craniotomies; other reconstructive techniques except EM or PF; subjects undergoing repair of spontaneous CSF leaks or encephaloceles.

Eligible subjects' basic data such as age at surgery, sex, final histology, time of follow-up, surgical procedure, repair technique, size of skull base defect, degree of frontal lobe sagging, and postoperative complications were recorded and analysed. The study protocol was approved by the local Ethics committee and adhered to the principles outlined in the Declaration of Helsinki. Informed consent was obtained from all living subjects included in the study.

Size of anterior cranial fossa defect and degree of frontal lobe sagging

According to the work published by Eloy et al. (3), an independent radiologist calculated the size of the anterior cranial fossa defect based on the bony defect identified on postoperative MRI or CT scans.

Also for the evaluation of frontal lobe sagging the same method proposed by Eloy et al. ${ }^{(3)}$ has been used. DICOM images from the preoperative and the last postoperative MRI or CT scan available has been analysed using $\operatorname{Horos}^{\circledast}$ (version 2.3.0 for $\operatorname{Mac}^{\circledR} 2015$,

Annapolis, MD, USA) image processing program. Using a paramedian image, a straight line was drawn from the nasion to the base of the sella turcica, which they defined the "nasion- sellar line". The position of the lowest aspect of the frontal lobe with respect to the nasion-sellar line was measured by tracing a normal from the lowest point of the frontal lobe to the nasionsellar line. This distance was measured on preoperative imaging (Xpre) and on the most recent postoperative image (Xpost). If the lowest aspect of the frontal lobe was below the nasion-sellar line, the distance was considered negative, otherwise positive. The degree of frontal lobe displacement $(\Delta)$ after surgery was calculated by subtracting Xpre from Xpost ( $\Delta=$ Xpost-Xpre). A positive value of $\Delta$ meant superior displacement of the frontal lobe, while a negative value of $\Delta$ meant inferior sagging of the brain after surgery.

Reconstruction procedures and postoperative management: three-layer technique

Before duraplasty, some preparatory steps are required. They must include: harvesting of the vascularized pedicled nasoseptal flap at the beginning of the surgery, if planned and oncologically adequate; appropriate exposure of the defect; undermining of the dura mater before its opening in order to create a pocket between the bone of the skull base and the dura mater; circumferential denudation of the mucosa surrounding the defect and smoothing of the defect edges to get a tensioactive effect for the graft or flap; dural resection until tumour free margins are obtained. We usually employ the ilio-tibial tract or fascia lata for the three layers reconstruction: the first layer is placed intradurally; the second one is placed intracranially but extradurally (this means that it is housed in the pocket previously created between the bone of the skull base and the dura mater); the third one is placed extradurally and extracranially and it can be replaced by the naso-septal flap or other mucosal lining, if it is available and oncologically safe. In case of benign lesions, the third layer is always made up by mucosa. The third layer is covered with strips of Surgicel (Ethicon Inc., Johnson and Johnson, Somerville, $\mathrm{NJ}$ ) even if it is the naso-septal flap, and fibrin glue is placed along the graft or flap margins ${ }^{(4,5)}$.

At the end of the surgery the nasal cavities are packed with Merocel nasal tampons for 48 hours and the subject is invited to bed rest with his/her head at 30 degrees for 3 days and to avoid any activity that could raise intracranial pressure such as straining or nose blowing for 1 month. Lumbar drains are not routinely used. A CT scan is performed generally on the $2 \mathrm{nd}-3 \mathrm{rd}$ day, if the patient is well and no complications arise before. After the removal of nasal tampons, we perform a daily endoscopic medication to exclude the presence of CSF leak. From 7 to 10 days the subject is kept on an antibiotic regimen and subcutaneous low molecular weight heparin injection are administered, according to the subject's comorbidities. He/she is placed on stool softener and a second-generation antihistamine for 1 month. We usually start nasal irrigation one week after surgery and, once the subject is discharged, we perform endoscopic medications 
Table 1. Comparison of general data between different groups. A positive value of $\Delta$ meant superior displacement of the frontal lobe, while a negative value of $\Delta$ meant inferior sagging of the brain after surgery.

\begin{tabular}{|c|c|c|c|c|c|c|}
\hline Subjects' groups & Males (\%) & Females (\%) & Mean Age (years) & $\begin{array}{l}\text { Mean Follow-up } \\
\text { (months) }\end{array}$ & $\begin{array}{l}\text { Mean skull base } \\
\text { defect size }\left(\mathrm{cm}^{2}\right)\end{array}$ & $\begin{array}{c}\text { Mean frontal lobe dis- } \\
\text { placement }(\Delta)(\mathrm{mm})\end{array}$ \\
\hline All subjects & $15(75 \%)$ & $5(25 \%)$ & $71,45 \pm 10,87$ & $18,3 \pm 21,5$ & $7,49 \pm 3,59$ & $-1,4 \pm 6,3$ \\
\hline PF group & $7(70 \%)$ & $3(30 \%)$ & $72,6 \pm 13,27$ & $15,6 \pm 19,5$ & $9,62 \pm 3,64$ & $-0,45 \pm 8,92$ \\
\hline EM group & $8(80 \%)$ & $2(20 \%)$ & $70,3 \pm 8,38$ & $21 \pm 24$ & $5,35 \pm 1,94$ & $-2,34 \pm 1,55$ \\
\hline
\end{tabular}

Table 2. Single subjects' data. A positive value of $\Delta$ meant superior displacement of the frontal lobe, while a negative value of $\Delta$ meant inferior sagging of the brain after surgery.

\begin{tabular}{|c|c|c|c|c|c|c|c|}
\hline Subject & Sex & $\begin{array}{c}\text { Age } \\
\text { (years) }\end{array}$ & $\begin{array}{l}\text { Surgical } \\
\text { Technique }\end{array}$ & Pathology & $\begin{array}{l}\text { Follow-up } \\
\text { (months) }\end{array}$ & $\begin{array}{l}\text { Skull Base defect } \\
\text { size }\left(\mathrm{cm}^{2}\right)\end{array}$ & $\begin{array}{l}\text { Frontal Lobe Dis- } \\
\text { placement }(\Delta)(\mathrm{mm})\end{array}$ \\
\hline 1 & M & 86 & PF & Osteosarcoma & 2 & 8,87 & 7,98 \\
\hline 2 & M & 85 & PF & SCC & 1 & 15,46 & $-16,79$ \\
\hline 3 & M & 72 & PF & ITAC & 1 & 12,42 & $-14,08$ \\
\hline 4 & $\mathrm{~F}$ & 75 & PF & Esthesioneuroblastoma & 39 & 5,23 & 2,29 \\
\hline 5 & M & 72 & $\mathrm{PF}$ & ITAC & 7 & 7,65 & 2,17 \\
\hline 6 & M & 58 & PF & Recurrent SNUC & 11 & 12,29 & $-1,5$ \\
\hline 7 & $\mathrm{~F}$ & 84 & $\mathrm{PF}$ & ITAC & 21 & 7,09 & 3,75 \\
\hline 8 & M & 69 & PF & ITAC & 11 & 13,76 & $-1,3$ \\
\hline 9 & $\mathrm{~F}$ & 44 & PF & Esthesioneuroblastoma & 60 & 4,98 & 0,92 \\
\hline 10 & M & 81 & PF & Olfactory groove meningioma & 3 & 8,49 & 12,03 \\
\hline 11 & M & 77 & EM & ITAC & 5 & 2,37 & $-2,46$ \\
\hline 12 & M & 52 & EM & ITAC & 4 & 7,72 & $-3,68$ \\
\hline 13 & M & 80 & EM & ITAC & 1 & 6,66 & $-0,95$ \\
\hline 14 & M & 79 & EM & ITAC & 17 & 2,27 & $-1,1$ \\
\hline 15 & M & 63 & EM & ITAC & 39 & 6,43 & $-0,83$ \\
\hline 16 & M & 67 & EM & ITAC & 42 & 5,3 & $-3,23$ \\
\hline 17 & M & 69 & EM & ITAC & 5 & 3,79 & $-0,83$ \\
\hline 18 & M & 74 & EM & ITAC & 18 & 6,99 & $-3,29$ \\
\hline 19 & $\mathrm{~F}$ & 72 & EM & Olfactory schwannoma & 4 & 5,23 & $-5,43$ \\
\hline 20 & $\mathrm{~F}$ & 70 & EM & Olfactory groove meningioma & 75 & 6,76 & $-1,61$ \\
\hline
\end{tabular}

ITAC: intestinal-type adenocarcinoma; SCC: squamous cell carcinoma; SNUC: sinonasal undifferentiated carcinoma.

of the nasal cavities every 2-3 weeks until complete healing.

Reconstruction procedures and postoperative management: pericranial flap

With the head fixed on a three-pin head holder, a coronal incision is performed and a galeal-pericranial flap is harvested. After performing a fronto-basal craniotomy and cranialization of the frontal sinuses, the dura mater of the anterior skull base is undermined from the underlying bone until reaching the point of intracranial infiltration of the tumor. The pathologic skull base, dura mater and the intracranial portion of the tumor are then resected "en bloc" until tumor free margins are obtained. In the end the defect of the anterior skull base is covered by the galeal- pericranial flap housed between the bone and the dura mater. We do not use any additional rigid support such as bone graft or wire mesh but normally we place an extradural extracranial layer of ilio-tibial tract or fascia lata endoscopically. Whenever possible and oncologically safe we place extradurally a mucosal lining (if available the naso-septal flap) in order to reduce healing process time and to improve residual nasal function. Empty spaces within reconstruction are usually managed using autologous fat. In case of benign lesions (e.g. meningioma) we use, as outer layer, naso-septal flap or any other mucosal lining. Surgicel and fibrin glue are used to seal the defect ${ }^{(6)}$. A rigid fixation is used to stabilize the frontal bone operculum. In order to prevent subcutaneous blood collection one or two drainages 


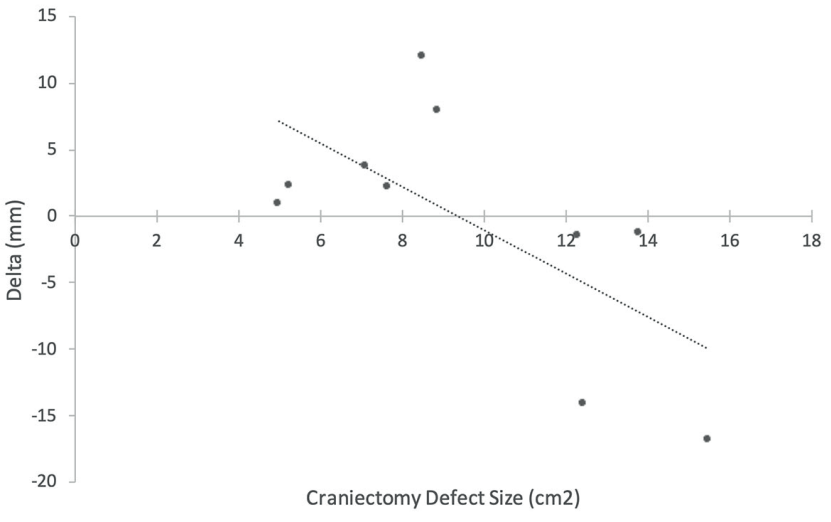

Figure 1. Correlation analysis between the size of craniectomies and changes in frontal lobe displacement $(\Delta)$ in subjects reconstructed with the $P F(r=-0,67 ; p<0,05)$.

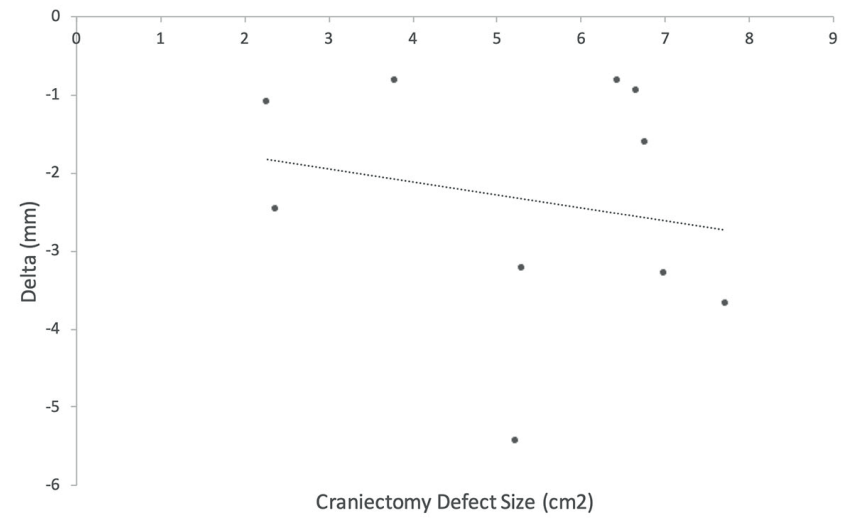

Figure 2. Correlation analysis between the size of craniectomies and changes in frontal lobe displacement $(\Delta)$ in subjects reconstructed with the EM technique $(r=-0,21 ; p>0,05)$. and a compressive bandage are placed.

The subject's post-operative management is comparable to the one already described for the Three-Layer Technique. The drainage is kept in place for two or three days.

\section{Statistical analysis}

Categorical variables are presented as percentage and continuous variables are presented as mean \pm standard deviation (SD). Differences between groups were tested by Mann-Whitney $\mathrm{U}$ test or Fisher exact test, respectively. Correlations were tested using Pearson correlation coefficient. Statistical analyses were performed using JMP Pro 11.2 software (SAS Institute Inc., Cary, NC, USA). A 2-sided $P<0,05$ was considered statistically significant.

\section{Results}

Twenty subjects met our inclusion and exclusion criteria over the period from June 2012 to December 2018 and were included in this analysis. They were 15 males (75\%) and 5 females (25\%), with a mean age of 71,45 years at surgery (range, 44/86 years); the mean follow-up period was $18,3 \pm 21,5$ months (ranging from 1 to 75 months). Ten subjects were reconstructed with PF while the other ten subjects were reconstructed with the EM technique (Table 1). The final histology of the removed neoplasms was the following: twelve intestinal-type adenocarcinomas (60\%), two esthesioneuroblastomas (10\%), two olfactory groove meningiomas (10\%), one osteosarcoma (5\%), one squamous cell carcinoma (5\%), one recurrent sinonasal undifferentiated carcinoma (5\%) and one olfactory schwannoma (5\%). We had no postoperative CSF leaks in any subject (Table 2).

Measurements of skull base defect size and postoperative frontal lobe sagging $(\Delta)$ are listed in Table 1 and 2 . The average skull base defect size was 5,35 $\pm 1,94 \mathrm{~cm}^{2}$ (range, 2,27/7,72 $\mathrm{cm}^{2}$ ) in subjects reconstructed with the EM technique and 9,62 $\pm 3,64$ $\mathrm{cm}^{2}$ (range, 4,98/15,46 $\mathrm{cm}^{2}$ ) in subjects reconstructed with the
PF and a significant difference was found between these two groups $(p<0,05)$.

All subjects reconstructed with the EM technique had an inferior displacement of the frontal lobe and the average $\Delta$ was $-2,34$ $\pm 1,55 \mathrm{~mm}$ (range, $-5,43 /-0,83 \mathrm{~mm}$ ). Four out of ten subjects reconstructed with the PF had a negative $\Delta$ and the average postoperative frontal lobe sagging was $-0,45 \pm 8,92 \mathrm{~mm}$ (range, $-16,79 / 12,03 \mathrm{~mm}$ ). These four subjects had the largest skull base defect size within their group $\left(>12 \mathrm{~cm}^{2}\right)$. We did not find a significant difference in frontal lobe sagging between the two groups $(p>0,05)$ due to the presence of subjects number two and three but, excluding these two subjects, we were able to identify a significant difference between these two groups $(p<0,05)$. The skull base defect size correlated with the degree of frontal lobe sagging $(r=-0,67 ; p<0,05)$ in subjects reconstructed with the PF (Figure 1), but the same correlation was not significant in the other group ( $r=-0,21 ; p>0,05)$ (Figure 2$)$ and when merging the two groups $(r=-0,37 ; p>0,05)$.

No significant difference was identified comparing subjects according to their sex, age, follow-up time and the execution of adjuvant radiotherapy.

\section{Discussion}

Recent technological and technical developments in skull base surgery have led another reliable reconstructive option to complement the pericranial flap in the reconstruction of postoperative anterior cranial fossa defects. As a matter of fact the EM technique has shown low incidence of postoperative CSF leak ${ }^{(7-9)}$, comparable to PF reconstruction. In our case series the EM technique had a good reliability, showing postoperative CSF leak in no case. Subjects who underwent reconstruction for spontaneous CSF leaks or encephaloceles were excluded because these defect sizes were generally smaller than defects created after tumor resection.

No studies with the aim to evaluate post-operative complicati- 

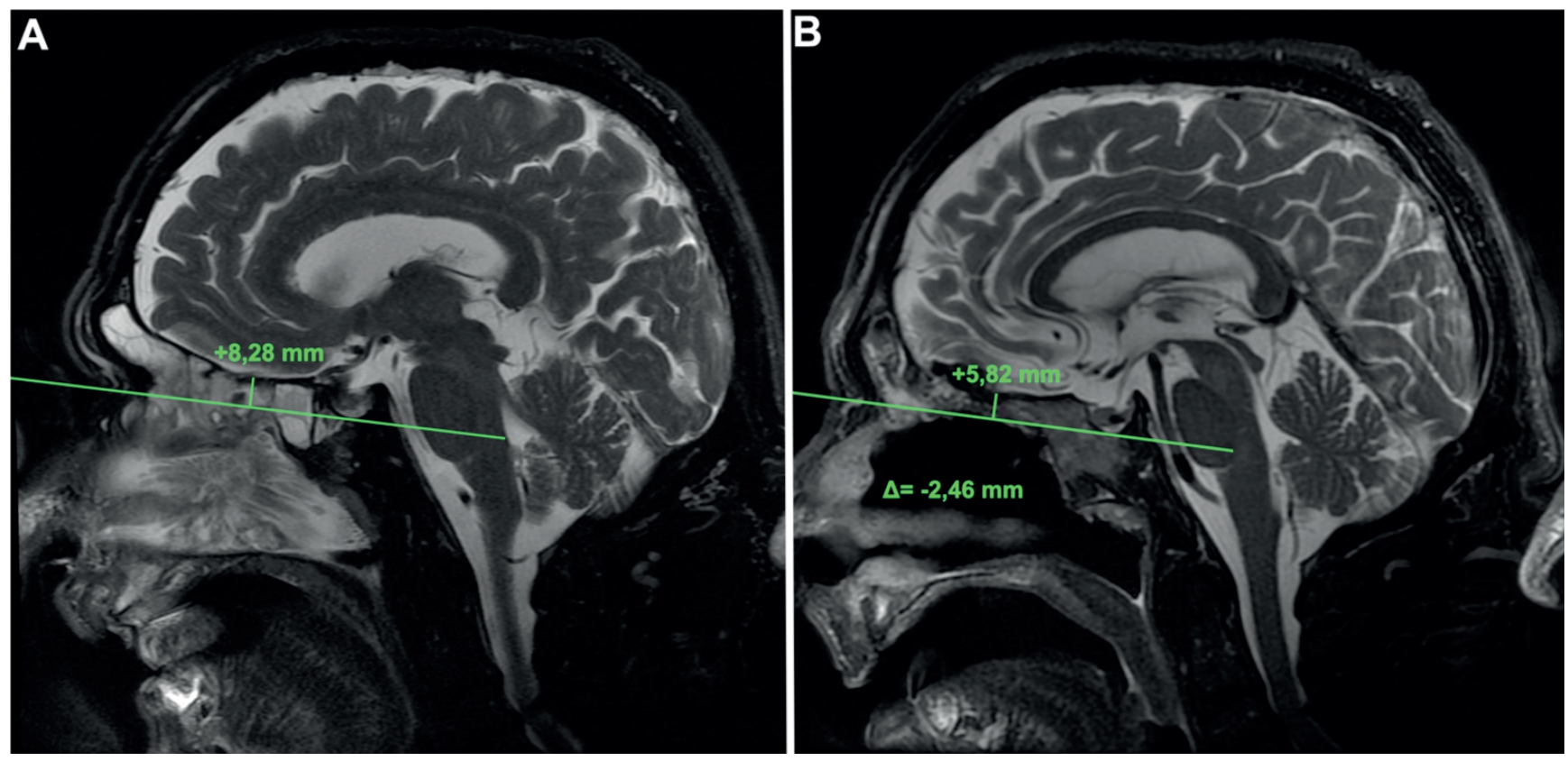

Figure 3. Sagittal preoperative (A) and postoperative (B) T2-weighted MRI showing an intestinal-type adenocarcinoma in subject \#11 that was resected using an endoscopic endonasal approach with subsequent repair of the skull base defect using the EM technique. Note the limited degree of brain sagging $(\Delta=-2,46 \mathrm{~mm})$.
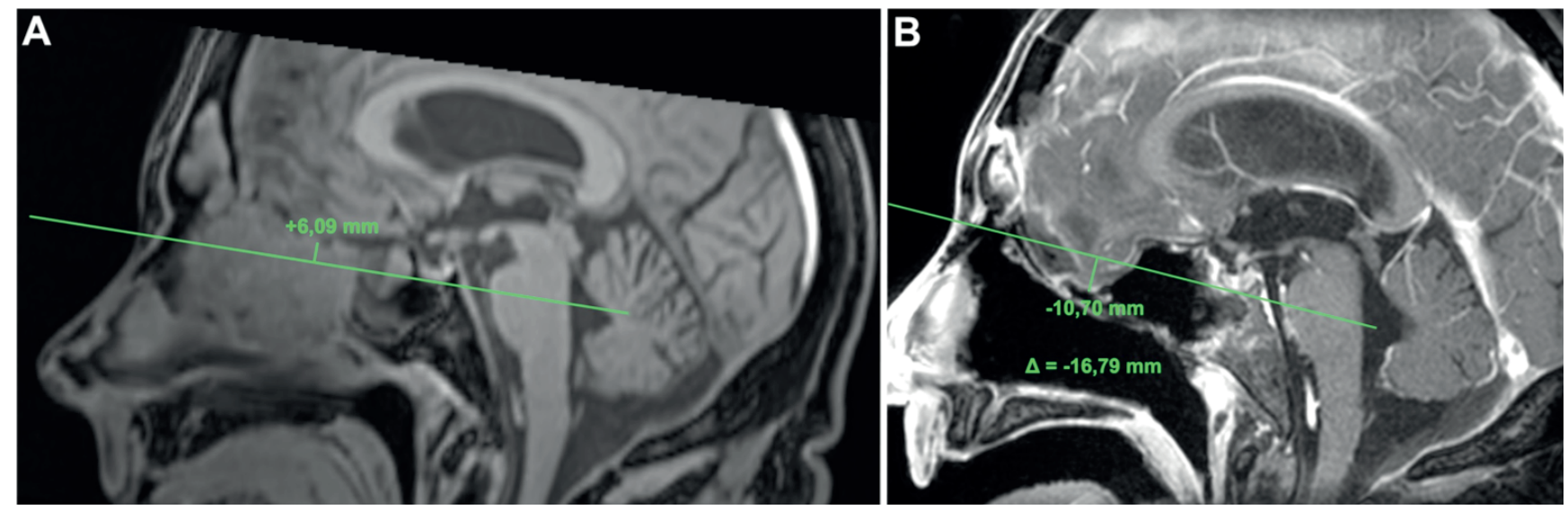

Figure 4. Sagittal preoperative (A) and postoperative (B) T1-weighted MRI showing a squamous cell carcinoma in subject \#2 that was resected using an external approach with subsequent repair of the skull base defect using the PF. Note the high degree of brain sagging $(\Delta=-16,79 \mathrm{~mm})$ that configures a frontal lobe falling.

ons in anterior skull base surgery, except one ${ }^{(3)}$, have ever evaluated the frontal lobe sagging ${ }^{(10,11)}$. To the best of our knowledge, this is the first work that analyses this postoperative outcome comparing it in two different surgical techniques.

In this work, we analysed and compared two different groups of subjects affected by benign or malignant neoplasms of the anterior cranial fossa taking into consideration the size of skull base defect and the degree of frontal lobe sagging after surgery. In these two groups we observed a significant difference in terms of size of skull base defect and this is justified by the choice of an external approach to manage larger tumors that could involve the cribriform plate, the lamina lateralis and the ethmoidal roof, but also the orbital roof. Since the dural resection was performed in close proximity of the edge of the bony defect, the resection size was calculated on the size of the bony defect.

Nevertheless we did not find a significant difference in frontal lobe sagging between the two groups due to the presence of subjects number two and three who had two of the worst outcomes and a short follow-up period because of their death. As a matter of fact, not considering these two subjects (for the very large skull base defects involving also a not minor part of the orbital roof associated with orbital exenteratio), we were able to identify a significant difference between the two groups. 
This means that if the skull base defect is not very large, the PF reconstruction determines a superior displacement of the brain while a reconstruction with the EM technique causes a frontal lobe sagging (Figure 3). On the contrary, if a very large defect should be managed, like in subjects two and three, in which also not a minor part of the orbital roof is removed in association also with orbital exenteratio, even the use of the PF does not seem to be sufficient to prevent frontal lobe sagging. This is probably related to the combination of 2 critical factors: very large skull base defect and very wide empty spaces below the reconstruction (and so the absence of any kind of support to the reconstruction). These subjects died due to postoperative severe complications. In these patients early post op radiological evaluation showed an important and rapid inferior frontal lobe displacement $(-16,79 \mathrm{~mm}$ and $-14,08 \mathrm{~mm}$, respectively), that we called "frontal lobe falling". The real impact of these modifications is unclear, but we do feel that it can be associated at least with some local brain abnormalities, since the brain exceeded 1 centimetre of sagging through the entire surface of the duraplasty (Figure 4). In these major resections, in which there's also a vast empty space below the reconstruction, it could be necessary to use some form of autologous rigid material or eventually a free flap ${ }^{(12)}$ in order to reinforce the PF duraplasty. Unfortunately the use of heterologous materials is burdened by some possible side effects like extrusion or infection ${ }^{(13)}$. A significant correlation exists between the skull base defect size and the brain sagging in the group of subjects reconstructed with PF: the larger the size of the skull base defect, the greater the degree of frontal lobe sagging. Analysing our data we identified as a "turning point" that limited the use of PF the measure of about $12 \mathrm{~cm}^{2}$ for skull base defect size. So if you plan to remove more than $12 \mathrm{~cm}^{2}$ of the anterior skull base we suggest thinking about the use of other rigid autologous or heterologous materials or free flaps to reinforce the PF. The same correlation was negative $(r=-0,21)$ but not significant $(p>0,05)$ in the EM group, so we can conclude that for small skull base defects the degree of frontal lobe sagging is not affected by the size of the resection, as already said by Eloy et al. ${ }^{(3)}$.

Eloy et al. ${ }^{(3)}$ showed a mild superior displacement of frontal lobe in $55,6 \%$ of their subjects reconstructed with the EM technique, while all our subjects reconstructed with the same technique showed a frontal lobe sagging. We think that this difference is due to the surgical technique and the use of different materials (fascia lata/fascia lata/naso-septal flap in our group VS fascia lata/implantable acellular dermal allograft/naso-septal flap in Eloy's group) but the small number of subjects and the short follow-up period do not allow us to draw any conclusion. Moreover they reported two subjects with cribriform defect size greater than $12 \mathrm{~cm}^{2}$ but one showed a superior displacement of frontal lobe $(2,2 \mathrm{~mm})$ and the other one a frontal lobe sagging
$(-1,9 \mathrm{~mm})$.

Battaglia et al. ${ }^{(14)}$ reported two cases of frontal lobe herniation identified between more than 500 cases of endoscopic anterior skull base reconstruction after malignant tumor resection, confirming that it is a rare complication of this kind of surgery. Nevertheless, we have to distinguish frontal lobe sagging, frontal lobe falling and frontal lobe herniation. Frontal lobe sagging is an inferior displacement less than $1 \mathrm{~cm}$ of the frontal lobe through the entire surface of the skull base defect; frontal lobe falling means an inferior displacement more than $1 \mathrm{~cm}$ of the brain through the entire surface of the skull base defect; frontal lobe herniation refers to an acute shift of cerebral tissue into the nose through the defect. The acuity of this last process, probably related to some form of brain oedema or eventually co-morbidities (i.e. sleep apnoea syndrome) makes it really a critical issue to be considered. Moreover, generally speaking, the degree of inferior displacement is greater in brain herniation than in brain sagging. We agree with Battaglia et al. ${ }^{(14)}$ that possible factors involved in the pathogenesis of these complications could be increased by intracranial pressure, obesity and obstructive sleep apnoea, not forgetting radionecrosis that could increase duraplasty weakness.

Furthermore a progressive increase of the degree of frontal lobe sagging should be taken into account when adjuvant radiotherapy is planned. In fact the inclusion of brain parenchyma into the radiation field can cause brain oedema and necrosis. For this reason, a strong collaboration between surgeons, radiologists and radiotherapists is mandatory to manage such complex cases $^{(14)}$.

Our work shows some limitations. The main one is intrinsic to all single institutional retrospective studies like ours: data are rarely collected according to the needs of the study and subjects are managed according to the clinical practice of that peculiar hospital, in a variable window of time. The small number of subjects, the short follow-up period and its high standard deviation represents other limitations.

Finally, we believe that larger experiences in multi-institutional prospective randomized controlled double-blinded studies and longer follow-up should be performed to confirm the importance of early identification of frontal lobe sagging or falling in order to prevent eventually major complications related to these conditions.

\section{Conclusion}

The EM technique and the PF reconstruction showed a good reliability for the closure of anterior skull base defects. Moreover the PF seemed to prevent frontal lobe sagging but, for very large skull base defects $(>12 \mathrm{~cm} 2)$, it could be useful to be combined with other autologous or heterologous materials to avoid the frontal lobe falling. 


\section{Acknowledgements}

There was no funding to declare.

\section{Authorship contribution}

GFmade substantial contributions to conception and design, acquisition of data, analysis and interpretation of data. He participated in drafting the article and revising it critically for important intellectual content and he gave final approval of the version to be submitted. SDS made substantial contributions to acquisition of data, analysis and interpretation of data. She participated in revising the article critically for important intellectual content and she gave final approval of the version to be submitted. DT made substantial contributions to conception and design, analysis and interpretation of data. He gave final approval of the version to be submitted. CC made substantial contributions to acquisition of data, analysis and interpretation of data. She participated in drafting the article and revising it critically for important intellectual content. VS made substantial contributions to acquisition of data, analysis and interpretation of data. She participated in drafting the article and revising it critically for important intellectual content. LCM made substantial contributions to acquisition of data, analysis and interpretation of data. She participated in drafting the article and revising it critically for important intellectual content. SB participated in revising the article critically for important intellectual content and he gave final approval of the version to be submitted. LB participated in revising the article critically for important intellectual content and he gave final approval of the version to be submitted. MS made substantial contributions to acquisition of data, analysis and interpretation of data. She participated in drafting the article and revising it critically for important intellectual content. ID made substantial contributions to conception and design, acquisition of data, analysis and interpretation of data. He participated in drafting the article and revising it critically for important intellectual content and he gave final approval of the version to be submitted.

\section{Conflict of interest}

The authors have no conflict of interest to declare.

\section{References}

1. Lund VJ, Stammberger $H$, Nicolai $P$ Castelnuovo P, Beal T, Beham A, et al. European position paper on endoscopic management of tumours of the nose, paranasal sinuses and skull base. Rhinol Suppl 2010;22:1-143

2. Borg A, Kirkman MA, Choi D. Endoscopic Endonasal Anterior Skull Base Surgery: A Systematic Review of Complications During the Past 65 Years. World Neurosurg 2016;95:383-91.

3. Eloy JA, Shukla PA, Choudhry OJ, Singh R, Liu JK. Assessment of frontal lobe sagging after endoscopic endonasal transcribriform resection of anterior skull base tumors: Is rigid structural reconstruction of the cranial base defect necessary? Laryngoscope 2012;122(12):2652-7.

4. Castelnuovo P, Battaglia P, Turri-Zanoni M Volpi L, Bignami M, Dallan I. Transnasal skull base reconstruction using a 3-d endoscope: our first impressions. J Neurol Surg Part B Skull Base 2012;73(2):85-9.

5. Muscatello L, Dallan I, Seccia V, Marchetti M, Sellari-Franceschini S, Lenzi R. Endoscopic endonasal craniotomy in the management of selected ethmoidal malignancies: The University of Pisa experience. J CranioMaxillofac Surg 2011;39(8):619-23.

6. Siniscalchi EN, Angileri FF, Mastellone P, Catalfamo L, Giusa M, Conti A, et al. Anterior skull base reconstruction with a galeal-pericranial flap. J Craniofac Surg 2007;18(3):622-5

7. Hadad G, Bassagasteguy L, Carrau RL,
Mataza JC, Kassam A, Snyderman $\mathrm{CH}$, et al. A novel reconstructive technique after endoscopic expanded endonasal approaches: vascular pedicle nasoseptal flap. Laryngoscope 2006;116(10):1882-6.

8. Kassam AB, Thomas A, Carrau RL, Snyderman $\mathrm{CH}$, Vescan A, Prevedello D, et al. Endoscopic reconstruction of the cranial base using a pedicled nasoseptal flap. Neurosurgery 2008;63(1 Suppl 1):ONS44-52; discussion ONS52-53.

9. Eloy JA, Kuperan AB, Choudhry OJ, Harirchian S, Liu JK. Efficacy of the pedicled nasoseptal flap without cerebrospinal fluid (CSF) diversion for repair of skull base defects: incidence of postoperative CSF leaks. Int Forum Allergy Rhinol 2012;2(5):397-401.

10. Kassam AB, Prevedello DM, Carrau RL, Snyderman CH, Thomas A, Gardner P, et al. Endoscopic endonasal skull base surgery: analysis of complications in the authors' initial 800 patients. J Neurosurg 2011;114(6):1544-68.

11. Harvey RJ, Parmar P, Sacks R, Zanation AM Endoscopic skull base reconstruction of large dural defects: A Systematic Review of Published Evidence. The Laryngoscope 2012;122(2):452-9.

12. Pangrazi PP, De Francesco F, Di Rienzo A, Riccio M. Adipofascial Radial Forearm Free Flap for Anterior Skull Base Reconstruction in Complicated Forebrain Oncological Surgery. J Craniofac Surg. 2019 Sep;30(6):1724-1729.

13. Gil Z, Abergel A, Leider-Trejo L, Khafif A,
Margalit N, Amir A, et al. A comprehensive algorithm for anterior skull base reconstruction after oncological resections. Skull Base Off J North Am Skull Base Soc Al 2007;17(1):25-37.

14. Battaglia P, Turri-Zanoni M, Castelnuovo P Prevedello DM, Carrau RL. Brain Herniation After Endoscopic Transnasal Resection of Anterior Skull Base Malignancies: Neurosurgery 2015;11:457-62

Giacomo Fiacchini, MD Otorhinolaryngology, Audiology and Phoniatrics Operative Unit Azienda Ospedaliero Universitaria Pisana Department of Surgical, Medical, Molecular Pathology and Emergency Medicine University of Pisa Via Paradisa 2 56124, Pisa Italy

Tel. +39-(0)50-997502

Fax. +39-(0)50-99 7515

E-mail:g.fiacchini@gmail.com 